\title{
Variações ecomorfológicas e de uso de habitat em Piabina argentea (Characiformes, Characidae) da bacia do rio das Velhas, Minas Gerais, Brasil
}

\author{
Cecília G. Leal ${ }^{1,2}$, Nara T. Junqueira ${ }^{2}$, Hersília de A. e $\operatorname{Santos}^{3} \&$ Paulo S. Pompeu $^{2}$
}

\footnotetext{
1. Programa de Pós-Graduação em Ecologia Aplicada, Universidade Federal de Lavras (UFLA).

2. Laboratório de Ecologia de Peixes, Departamento de Biologia, Universidade Federal de Lavras (UFLA), 37200-000 Lavras, Minas Gerais, Brasil. (c.gontijoleal@gmail.com; naratadini@yahoo.com.br; pompeu@dbi.ufla.br)

3. Departamento de Engenharia Civil, Centro Federal de Educação Tecnológica de Minas Gerais (CEFET), Campus II, Av. Amazonas, 7675, Nova Gameleira, 30510-000 Belo Horizonte, Minas Gerais, Brasil. (hsantos@ civil.cefetmg.br)
}

\begin{abstract}
Ecomorphological and habitat use variations in Piabina argentea (Characiformes, Characidae) from Velhas River basin, Minas Gerais, Brazil. This study aimed to investigate the local and regional patterns of habitat use by Piabina argentea Reinhardt, 1867 in four different rivers from the Velhas River basin. Water velocity, depth and substrate were measured at each sampling site. Ecomorphological analysis was performed using 17 ecomorphological attributes and 40 specimens from each river. Although the populations overlapped in morphological space, Canonical Discriminant Analysis showed that they were statistically different, mainly the population from the Velhas River. The most important attributes in the analysis were compression index, relative body depth, and index of ventral flattening. The local patterns of habitat use were not similar between all rivers, but in general, the regional pattern was slow flowing habitat with 20 to $40 \mathrm{~cm}$ and 60 to $80 \mathrm{~cm}$ of depth and sand, silte+clay and leave banks as substrate. Considering the physical characteristics of each river and the pattern of regional habitat use, a great amount of the species requirements was observed in the four rivers. Nevertheless, a silted river like the Velhas seems to have higher water velocity, shallower habitat and fine particle substrate, which fulfill most of the species habitat requirements.
\end{abstract}

KEYWORDS. Ecomorphology, morphological overlap, intraspecific variation, São Francisco River basin.

RESUMO. O presente estudo teve como objetivo investigar os padrões local e regional de uso de habitat de Piabina argentea Reinhardt, 1867 em quatro diferentes rios da bacia do rio das Velhas. Os habitat amostrados foram caracterizados quanto à velocidade da água, profundidade e tipo de substrato. Para a análise ecomorfológica, foram calculados 17 atributos ecomorfológicos de 40 exemplares de cada rio. Embora estas populações tenham se sobreposto no espaço ecomorfológico, a Análise Discriminante Canônica mostrou haver diferença significativa entre elas, principalmente da população do rio das Velhas em relação às demais. A separação se deu em termos do índice de compressão, altura relativa do corpo e índice de achatamento ventral. Os padrões locais de seleção de habitat não foram congruentes em todos os rios, mas em geral, houve predomínio do padrão regional: habitat lênticos, profundidade entre 20 e $40 \mathrm{~cm}$ e 60 e $80 \mathrm{~cm}$ e substrato areia, silte + argila e banco de folhas. Considerando as características físicas de cada rio e o padrão regional da espécie, a maior parte dos seus requerimentos de habitat é contemplada nos quatro rios. Entretanto, um corpo d'água assoreado como o trecho do rio das Velhas, tende a ter maiores velocidades da água, menores profundidades e substrato finos, o que atende em parte à seleção de habitat da espécie estudada.

PALAVRAS-CHAVE. Ecomorfologia, sobreposição morfológica, variação intraespecífica, bacia do rio São Francisco

O formato corporal é um importante componente do fenótipo de um organismo, influenciando sua eficiência de forrageamento, desempenho locomotor, vulnerabilidade à predação e sucesso reprodutivo (GuILl et al., 2003). Em peixes, as características morfológicas das espécies podem refletir aspectos ecológicos importantes e dar indicativos de seus hábitos e adaptações aos ambientes onde vivem (GATZ, 1979a; Watson \& BALON, 1984; Winemiller, 1991). Desta forma, a morfologia do indivíduo pode determinar os limites de seu desempenho e estabelecer a amplitude de recursos que é capaz de explorar (WAINWRight, 1994). Dentro desse limite, os indivíduos tendem a ocupar os habitat aos quais estão mais adaptados, sendo geralmente intensa a relação entre a forma do corpo das espécies e determinadas variáveis ambientais, como velocidade da água, profundidade e tipo de substrato (GoRMAN \& KarR, 1978; Grossman \& De Sostoa, 1994; Jowett \& RichARDSON, 1995; GroSSMAN et al., 1998; LAMOUROUX et al., 1999; ALLEN, 2000; VADAS JúNIOR \& ORTH, 2000; BlANCK et al., 2007; LEAL et al., 2011).

Ambientes aquáticos geralmente apresentam grande variabilidade espacial e temporal de seus componentes bióticos e abióticos (LOWE-McCoNNELL, 1987). Desta forma, a diversificação morfológica intraespecífica é um fator importante para a sobrevivência de uma espécie em determinado ecossistema, pois possibilita maior capacidade de adaptação às diferentes condições ambientais, ao passo que um único morfótipo não deve oferecer o melhor desempenho para uma espécie em todas as situações (PakKasmaA \& PiIronen, 2001). Regimes seletivos diferentes podem gerar e manter diversidade morfológica que, por sua vez, pode ser resultado de diferenças genéticas ou plasticidade fenotípica (Robinson \& Wilson, 1994; Travis, 1994). Por outro lado, o fluxo gênico e a migração de indivíduos, devem restringir e limitar a diversificação adaptativa de populações em ambientes distintos (LANGERHANS et al., 2003).

A diversidade morfológica intraespecífica de peixes é bem documentada na literatura (RoBINSON \& Wilson, 1994; SMith \& Skúlason, 1996; Brinsmead \& Fox, 2002; LANGerhans et al., 2003; Neves \& MonTeIro, 2003), principalmente em relação às variações do caráter lótico ou lêntico dos ambientes. $\mathrm{O}$ entendimento de conceitos ecológicos e das relações de forma e função entre os caracteres morfológicos 
e aspectos do ambiente permitem o amplo uso da ecomorfologia em estudos ecológicos e evolutivos (Cunico \& Agostinho, 2006). Entretanto, sua aplicação como ferramenta para avaliar as consequências das alterações dos habitat por atividades antrópicas, ainda é pouco difundida. Sabendo-se que a morfologia das espécies pode estar relacionada à ocupação espacial (Gatz, 1979b; CAsatti \& CASTro, 2006; Leal et al., 2011), modificações nas características físicas e químicas dos habitat podem propiciar alterações nos padrões morfológicos de populações e assembleias de peixes (Cunico \& Agostinho, 2006).

Piabina argentea Reinhardt, 1867, popularmente conhecida como piaba, é uma espécie de pequeno porte que tem como localidade-tipo o rio das Velhas em Minas Gerais. Distribui-se amplamente pela América do Sul, com registro para as bacias dos rios alto Paraná, São Francisco, Itapicuru, Paraíba e Itapemirim (Lima et al., 2003). Na bacia do rio das Velhas, pode ser encontrada em diferentes ambientes, incluindo a calha principal e seus tributários (Alves \& Pompeu, 2001). Sendo uma espécie abundante e de ampla distribuição na referida bacia, o presente estudo teve como objetivo responder as seguintes perguntas: (i) o tipo de habitat explorado por $P$. argentea é o mesmo entre diferentes rios dessa mesma bacia? (ii) o tipo de habitat varia de acordo com as classes de tamanho da espécie? (iii) populações de $P$. argentea encontradas em diferentes rios diferem ecomorfologicamente? (iv) os padrões locais de uso de habitat relacionam-se às características ecomorfológicas dos indivíduos?

A hipótese testada no presente trabalho pressupõe que a ecomorfologia de $P$. argentea reflete a maneira com que a espécie explora o habitat. Desta forma, espera-se que rios mais degradados e com consequente perda e modificação de habitat apresentem populações ecomorfologicamente distintas dos rios mais preservados da mesma bacia.

\section{MATERIAL E MÉTODOS}

Área de estudo. O rio das Velhas, com área de drenagem de $27.867 \mathrm{~km}^{2}$, estende-se por $761 \mathrm{~km}$ (CETEC, 1983; Codevasf, 1986), desde sua nascente, próximo à região metropolitana de Belo Horizonte (RMBH), até sua foz, no rio São Francisco (Fig. 1). Dentre as principais atividades responsáveis por promover intensa modificação de suas condições ambientais estão a ocupação irregular das margens, o uso da água para abastecimento de diferentes cidades e o despejo de esgoto doméstico provindo de diversos municípios localizados ao longo de seu leito (ALVES \& PoMPEU, 2005).

No presente estudo, as coletas foram realizadas no rio Cipó, próximo ao município de Presidente Juscelino (18 $8^{\circ} 41^{\prime} 7.1$ 'S, 43॰59'48.7' W; $576 \mathrm{~m}$ ), no rio Curimataí, próximo ao município de Augusto de
Lima (1759’33.3”S, 44¹0’48.2”W; $543 \mathrm{~m})$, no rio Pardo Grande, próximo ao município de Santo Hipólito (18¹3'43.3"S, 44¹3'3.1'W; $516 \mathrm{~m}$ ) e também no próprio rio das Velhas, próximo ao município de Rio Acima (2006'01.44'S, 4347'35.85'W; 742 m) (Fig. 1). O rio das Velhas, no ponto amostrado, apresentase assoreado e degradado, enquanto que os tributários podem ser considerados trechos de referência na bacia, por abrigarem uma ictiofauna relativamente rica $\mathrm{e}$ possuírem maior integridade física do que a calha principal (PomPEu et al., 2005).

Coleta de dados. Os peixes foram capturados durante o dia, na estação seca, em setembro de 2007. Para captura dos espécimes foram utilizadas peneiras de tela mosquiteiro ( $80 \mathrm{~cm}$ de diâmetro, $1 \mathrm{~mm}$ de malha) e redes de arrasto $(6 \mathrm{~m}$ de comprimento, $5 \mathrm{~mm}$ de malha), sendo estimada a área de cada habitat $\left(\mathrm{em} \mathrm{m}^{2}\right)$. Em cada rio, as coletas aconteceram em um trecho de $500 \mathrm{~m}$ durante dois dias. Pelo menos 20 habitat foram amostrados em cada rio, exceto no rio Cipó, onde só foi possível amostrar 12. Um habitat foi definido como em LEAL et al. (2011): ambiente homogêneo em relação ao substrato, velocidade da água e profundidade (ex. corredeira rasa de cascalho, praia de areia e profundidade

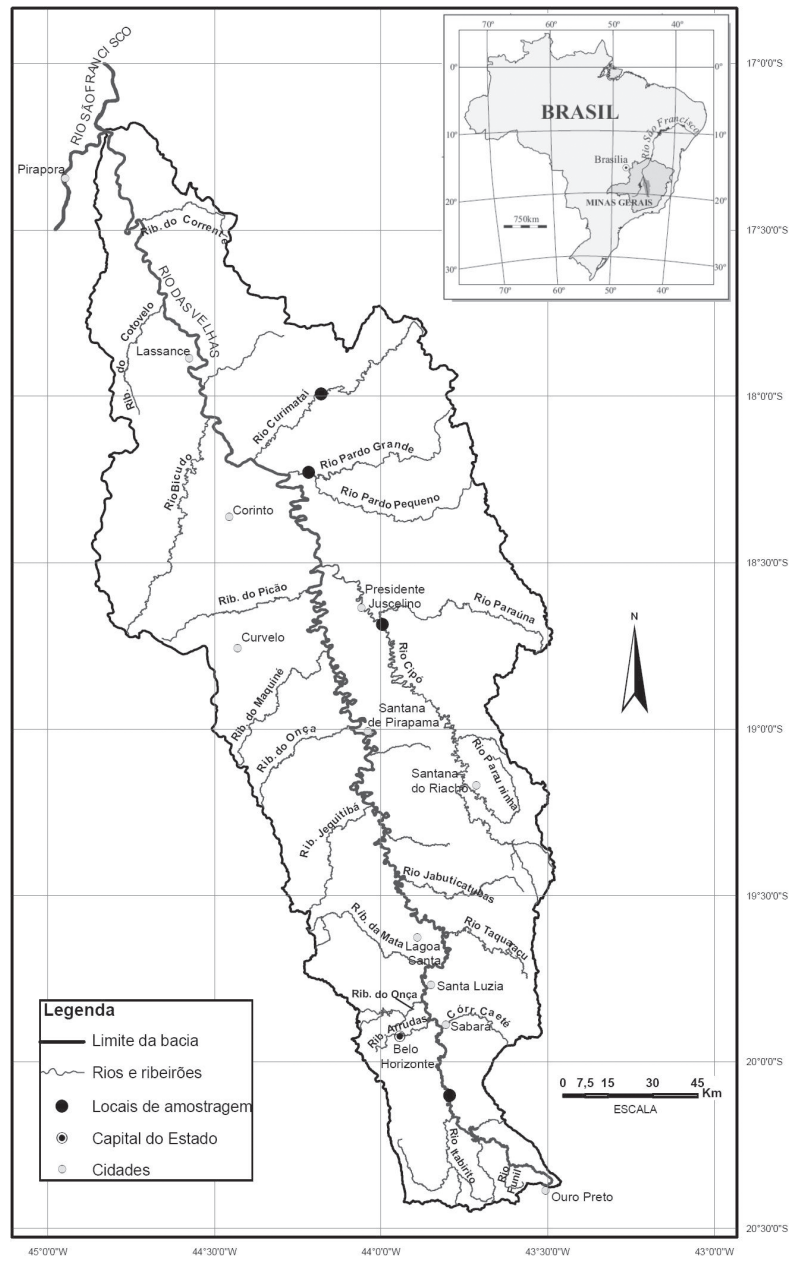

Fig. 1. Bacia do rio das Velhas, sudeste do Brasil. Trechos amostrados em setembro de 2007: rios das Velhas, Cipó, Pardo Grande e Curimataí. 
intermediária, remanso fundo de silte+argila), e foi amostrado com o petrecho que melhor se adequou às suas características e pelo tempo necessário para não mais capturar indivíduos. Os peixes coletados foram etiquetados por habitat, fixados em solução de formol $10 \%$ e conservados em solução de álcool $70^{\circ}$ GL. Exemplares testemunho foram depositados na Coleção Ictiológica do Núcleo de Pesquisas em Limnologia, Ictiologia e Aquicultura (Nupélia) (NUP7270; 20 exemplares; 36,6 - 44,54 mm de comprimento padrão).

Para caracterização dos habitat amostrados, a profundidade e a velocidade da água (medida a $60 \%$ da profundidade) foram obtidas com o molinete do tipo turbina, sendo seus valores separados por classes. Uma amostra de substrato foi recolhida para análise granulométrica em laboratório, e classificada em uma das seguintes categorias: silte + argila $(<0,063 \mathrm{~mm})$, areia $(0,063 \mathrm{~mm}$ a $<2 \mathrm{~mm})$, cascalho $(2 \mathrm{~mm}$ a $<16$ $\mathrm{mm})$, seixo (16 $\mathrm{mm}$ a $<300 \mathrm{~mm})$ e matacão $(\geq 300 \mathrm{~mm})$ (Suguio,1973; modificado por Callisto \& Esteves, 1996) e banco de folhas e raiz na margem.

A caracterização dos rios em termos das variáveis profundidade e velocidade média da água foi realizada em um trecho de $500 \mathrm{~m}$ subdividido em 25 seções, com 20 m de distância entre si. Dentro de cada seção, velocidade e profundidade foram medidas a $20 \%, 50 \%$ e $80 \%$ de distância da margem, sendo que a velocidade, também nesse caso, foi obtida a $60 \%$ da profundidade.

A fim de evitar variação produzida por diferenças ontogenéticas, as medidas morfológicas foram tomadas de 40 indivíduos de cada rio, com a mesma amplitude de comprimento padrão (entre 15 e $55 \mathrm{~cm}$ ). Estes indivíduos foram coletados em locais com velocidade da água entre 0 e $0,5 \mathrm{~m} / \mathrm{s}$. Todos os indivíduos foram medidos com paquímetro digital $(0,01 \mathrm{~mm}$ de precisão), sendo tomadas 16 medidas morfológicas lineares e cinco medidas de área de cada indivíduo. A medida das áreas foi feita estendendo e desenhando as nadadeiras e o corpo dos peixes em papel milimetrado. Em seguida foram calculados 17 atributos ecomorfológicos para a espécie: índice de compressão (IC), altura relativa do corpo (AR), índice de achatamento ventral (IAV), posição relativa dos olhos (PRO), posição da boca (PB), comprimento relativo da cabeça (CRC), comprimento relativo do pedúnculo caudal (CRPC), índice de compressão do pedúnculo caudal (ICPC), razão aspecto nadadeira caudal (RANC), razão aspecto da nadadeira peitoral (RANPT), razão aspecto da nadadeira pélvica (RANPL), comprimento relativo da nadadeira peitoral (CRNPT), comprimento relativo da nadadeira pélvica (CRNPL), área relativa de nadadeira caudal (ARNC), área relativa da nadadeira peitoral (ARNPT), área relativa da nadadeira pélvica (ARNPL), área relativa da nadadeira dorsal (ARND). A descrição detalhada do cálculo e a interpretação ecológica destes atributos podem ser encontradas em HoRA (1930), GosLINE (1971), GATZ (1979b), Watson \& Balon (1984), Freire \& Agostinho (2001), CASATTI \& CASTRO (2006).
Análise dos dados. Para verificar a ordenação dos indivíduos de $P$. argentea de cada rio no espaço morfológico, foi realizada uma Análise dos Componentes Principais (PCA) a partir de uma matriz de correlação considerando os valores sem transformação dos 17 atributos ecomorfológicos. Eixos com autovalor acima de um foram retidos para interpretação (GATZ 1979a; WATSON \& BALON, 1984). Para avaliar se existem diferenças significativas na ocupação do espaço ecomorfológico entre as populações de cada rio, foi conduzida uma Análise de Variância (ANOVA) seguida pelo Teste de Tukey considerando os grupos de escores de cada rio resultantes dos dois primeiros eixos da PCA. Nesta análise, o eixo 1 (PCA1) e o eixo 2 (PCA2) foram considerados separadamente. Essas análises foram realizadas através do programa Statistica 6.0 (STATSofT, 2001).

Para avaliar se as populações de $P$. argentea são diferentes ecomorfologicamente entre os rios, foi aplicada uma Análise Discriminante Canônica (DCA) passo-a-passo Backward considerando a configuração padrão do programa Statistica 6.0 (STATSOFt, 2001) e como grupos definidos a priori as populações de cada rio da bacia do rio das Velhas. A análise, mais robusta do que a PCA em identificar tendências de segregação entre grupos, começa com todos os atributos ecomorfológicos no modelo e a cada passo exclui aquele com maior valor de $\mathrm{F}$ e menor tolerância. Por fim, ficam retidos no modelo apenas os atributos que mais contribuem para a discriminação dos grupos.

Para testar a hipótese nula de que não existe correlação entre a morfologia das populações e o uso de habitat, foi realizado o teste de Mantel entre a matriz de distância ecomorfológica entre pares de indivíduos (distância Euclidiana) e a matriz de uso de habitat (sendo o valor "1" atribuído aos pares de indivíduos que ocupam o mesmo rio e o valor "0" àqueles que ocupam rios distintos) através do programa $\mathrm{R}$ ( $\mathrm{R}$ DEVELOPMENT Core Team, 2012).

Para avaliar a seleção de habitat de $P$. argentea na bacia do rio das Velhas, a frequência de uso de habitat pelas populações (número de indivíduos em cada classe de velocidade da água, profundidade e tipo de substrato) foi dividida pela sua disponibilidade (área amostrada), obtendo-se assim a densidade de indivíduos. A seleção por determinado habitat é detectada quando um organismo o utiliza em maior proporção do que ele está disponível no ambiente (Rosenfeld, 2003). Esta avaliação foi feita também para cada uma das classes de comprimento padrão, em relação à velocidade da água, profundidade e tipo de substrato.

\section{RESULTADOS}

O rio Cipó caracterizou-se por menor velocidade da água e maior profundidade (Tab. I), leito praticamente contínuo, com praias restritas às margens e um pequeno 
Tab. I. Média e desvio padrão da largura, profundidade, velocidade da água e área de drenagem (considerada a área a montante do ponto de amostragem) dos rios da bacia do rio das Velhas, sudeste do Brasil, setembro de 2007.

\begin{tabular}{|c|c|c|c|c|}
\hline Rios & Largura (m) & Profundidade (m) & Velocidade da água $(\mathrm{m} / \mathrm{s})$ & Área de drenagem $\left(\mathrm{km}^{2}\right)$ \\
\hline Cipó & $39,57 \pm 5$ & $1,88 \pm 0,78$ & $0,30 \pm 0,40$ & 1411,81 \\
\hline Curimataí & $23,03 \pm 4$ & $0,67 \pm 0,44$ & $0,68+0,90$ & 1417,61 \\
\hline Pardo Grande & $39,93 \pm 6$ & $0,55 \pm 0,37$ & $0,72 \pm 0,59$ & 1986,42 \\
\hline Velhas & $24,59 \pm 6$ & $1,33 \pm 0,81$ & $1,53 \pm 1,04$ & 2101,56 \\
\hline
\end{tabular}

trecho encachoeirado de margem a margem. $\mathrm{O}$ rio Pardo Grande e o rio Curimataí foram os mais rasos e com valores intermediários de velocidade da água (Tab. I). A maior parte do trecho estudado no Pardo Grande caracterizou-se por praias de areia, sendo uma porção desse trecho caracterizada por habitat rasos (até $20 \mathrm{~cm}$ ) e substrato composto predominantemente por cascalhos e seixos, onde havia corredeiras e poções. Por outro lado, o rio Curimataí apresentou todos os tipos de substrato, exceto silte+argila, destacando-se pelas diversas corredeiras com blocos de pedra e também pela abundância de bancos de folhas e vegetação com raízes submersas. O rio das Velhas, com maior velocidade da água e com a segunda maior profundidade, apresentou principalmente substrato fino como silte+argila e areia, sendo a maior parte deste substrato originário das minerações à montante ou do material de construção e lixo doméstico particulado do seu entorno.

No rio das Velhas foi registrada a maior densidade de $P$. argentea $\left(0,94\right.$ indivíduos $\left./ \mathrm{m}^{2}\right)$ e a maior abundância de indivíduos (273), seguido pelos rios Cipó $\left(0,57\right.$ indivíduos $\left./ \mathrm{m}^{2} ; 133\right)$, Curimataí $(0,36$ indivíduos/ $\left.\mathrm{m}^{2} ; 100\right)$ e Pardo Grande $\left(0,33\right.$ indivíduos $\left./ \mathrm{m}^{2} ; 197\right)$ (Figs 2-5). Pardo Grande e Cipó apresentaram maior abundância de indivíduos de 30 a $35 \mathrm{~cm}$ de comprimento padrão. No rio Curimataí, a classe mais frequente foi de 25 a $30 \mathrm{~cm}$, enquanto no rio das Velhas os indivíduos mais frequentes foram menores, entre 20 e $25 \mathrm{~cm}$.

Embora os seis primeiros eixos tenham apresentado autovalores maiores do que um, apenas os dois primeiros apresentaram padrões com interpretações ecomorfológicas relevantes e foram responsáveis pela maior parte da variância, 29,3\% de explicação (Tab. II). O primeiro componente explicou $18,8 \%$ e os atributos que mais contribuíram negativamente para sua formação foram área relativa da nadadeira pélvica e comprimento relativo da nadadeira pélvica, enquanto que comprimento relativo do pedúnculo caudal, razão aspecto da nadadeira peitoral, razão aspecto da nadadeira pélvica, índice de achatamento ventral e índice de compressão do pedúnculo caudal contribuíram positivamente para a formação eixo. Este eixo discriminou indivíduos menos achatados ventralmente (menor IAV), com nadadeiras pélvicas e peitorais maiores (maiores ARNPL e ARNPT) e mais largas do que longas (menores RANPL e RANPV), pélvicas mais longas em relação ao corpo (maior CRNPL) e pedúnculo caudal mais alto e curto (menores ICPC e CRPC), dos indivíduos mais achatados ventralmente, com nadadeiras pares menores e mais longas do que largas, pélvicas mais curtas em relação ao

Tab. II. Contribuição dos 17 atributos ecomorfológicos nos dois primeiros eixos do PCA, para Piabina argentea Reinhardt, 1867 coletada nos rios Cipó, Curimataí, Pardo Grande e Velhas, bacia do rio das Velhas, sudeste do Brasil, setembro de 2007. Valores maiores que -0,3 e menores que 0,3 estão representados apenas com sinal positivo ou negativo; valores maiores que $-0,5$ e 0,5 estão destacados em negrito.

\begin{tabular}{|c|c|c|c|c|c|c|}
\hline \multirow{2}{*}{ Atributos ecomorfológicos } & \multicolumn{6}{|c|}{ Eixos } \\
\hline & PCA 1 & PCA2 & PCA 3 & PCA 4 & PCA 5 & PCA 6 \\
\hline Índice de compressão & + & + & - & 0,51 & 0,56 & - \\
\hline Altura relativa & - & 0,52 & $-0,54$ & 0,31 & + & + \\
\hline Índice de achatamento ventral & 0,52 & $-0,34$ & + & - & - & - \\
\hline Posição relativa dos olhos & $-0,42$ & + & + & - & + & + \\
\hline Posição da boca & + & - & + & - & 0,66 & + \\
\hline Comprimento relativo da cabeça & - & $-0,36$ & - & + & 0,37 & + \\
\hline Comprimento relativo do pedúnculo caudal & 0,55 & - & + & $-0,31$ & + & + \\
\hline Índice de compressão do pedúnculo caudal & 0,52 & - & - & + & + & 0,42 \\
\hline Razão aspecto da nadadeira caudal & - & + & 0,68 & 0,39 & + & + \\
\hline Razão aspecto da nadadeira peitoral & 0,54 & - & + & + & $-0,34$ & - \\
\hline Razão aspecto da nadadeira pélvica & $\mathbf{0 , 5 3}$ & - & + & + & + & $-0,39$ \\
\hline Comprimento relativo da nadadeira peitoral & - & $-0,44$ & - & 0,51 & - & 0,48 \\
\hline Comprimento relativo da nadadeira pélvica & $-0,58$ & - & 0,32 & + & - & + \\
\hline Área relativa da nadadeira caudal & - & $-0,56$ & $-0,49$ & $-0,38$ & + & + \\
\hline Área relativa da nadadeira peitoral & $-0,53$ & $-0,30$ & $-0,31$ & + & - & - \\
\hline Área relativa da nadadeira pélvica & $-0,74$ & - & + & + & - & - \\
\hline Área relativa da nadadeira dorsal & $-0,45$ & $-0,41$ & + & - & + & $-0,38$ \\
\hline Autovalor & 3,2 & 1,8 & 1,5 & 1,5 & 1,3 & 1,1 \\
\hline Proporção da variação (\%) & 18,8 & 10,5 & 8,9 & 8,6 & 7,7 & 6,4 \\
\hline Variação cumulativa (\%) & & 29,3 & 38,2 & 46,8 & 54,5 & 60,8 \\
\hline
\end{tabular}



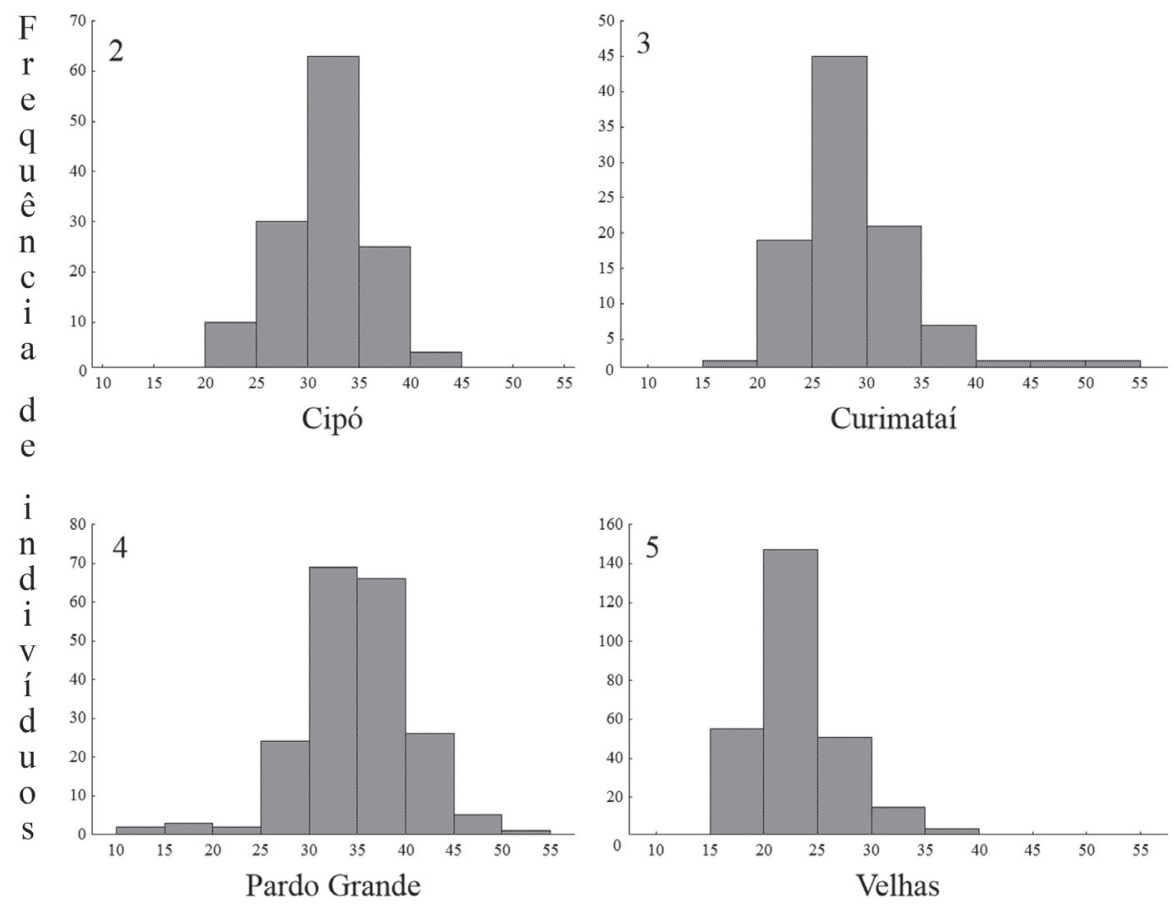

Classes de comprimento padrão $(\mathrm{mm})$

Figs. 2-5. Distribuição dos indivíduos de Piabina argentea Reinhardt, 1867 em classes de comprimento padrão (mm), para os quatro rios amostrados na bacia do rio das Velhas, setembro de 2007.

corpo e pedúnculo caudal mais baixo e longo. O segundo eixo reteve $10,5 \%$ da variância, sendo a área da nadadeira caudal relacionada negativamente e a altura relativa do corpo, positivamente. $\mathrm{O}$ gradiente evidenciado pela análise foi de indivíduos com grande área da nadadeira caudal e corpo mais baixo, a indivíduos com nadadeira pequena e corpo mais alto.
No espaço ecomorfológico, as quatro populações apresentaram grande sobreposição, embora, nos extremos de sua distribuição os grupos sejam diferentes (Fig. 6). Considerando o primeiro eixo da análise (PCA1), os indivíduos dos rios Cipó, Curimataí e Pardo Grande encontram-se principalmente próximo ao zero e à esquerda deste. Para o rio das Velhas, a maioria dos

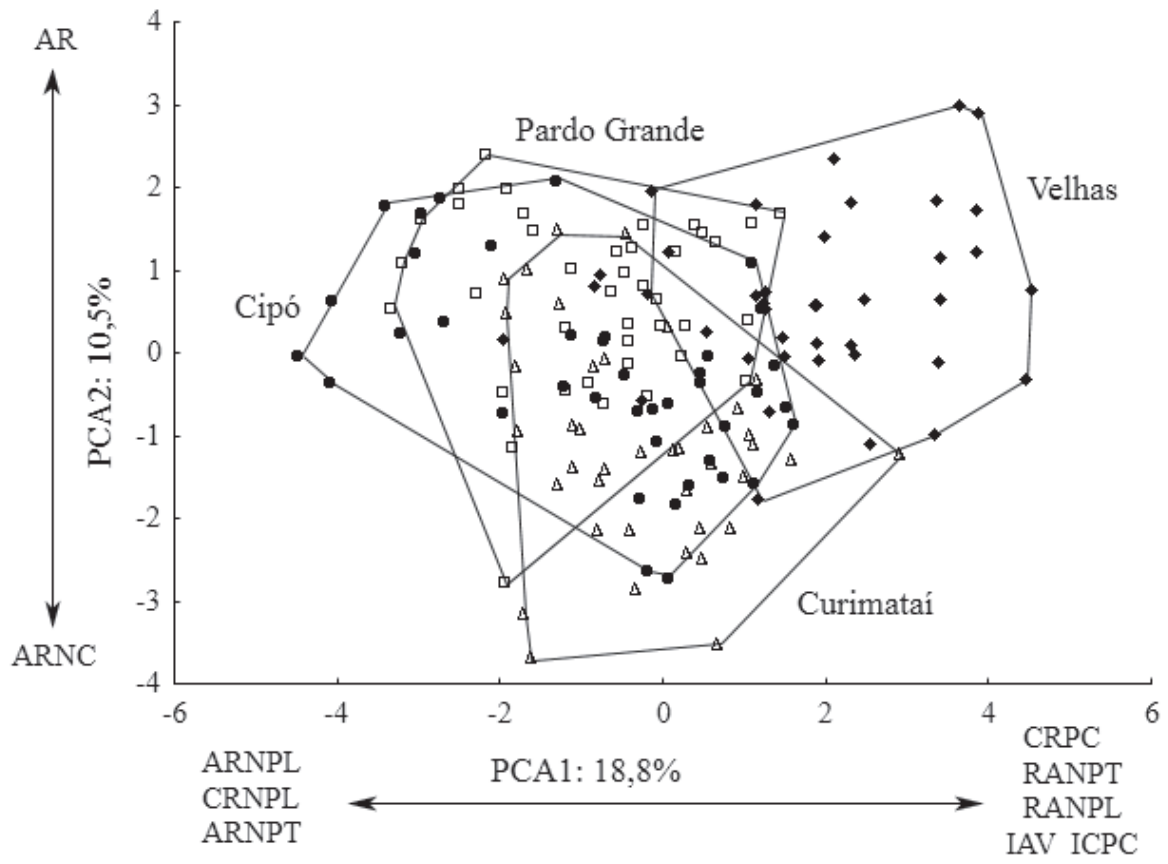

Fig. 6. Projeção dos dois primeiros eixos da Análise dos Componentes Principais baseada em atributos ecomorfológicos dos exemplares de Piabina argentea Reinhardt, 1867 coletados nos rios Cipó, Curimataí, Pardo Grande e Velhas, bacia do rio das Velhas, sudeste do Brasil, setembro de 2007 


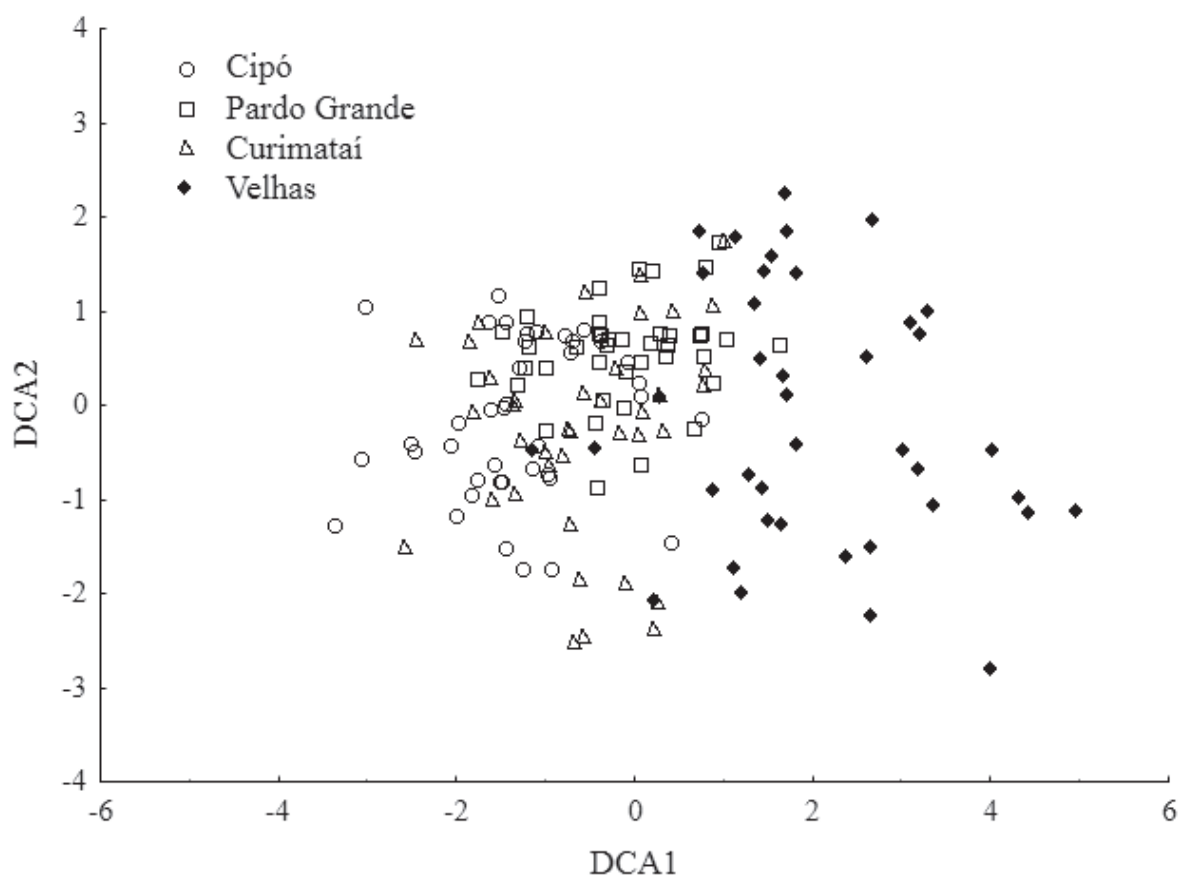

Fig. 7. Projeção dos escores canônicos individuais dos exemplares de Piabina argentea Reinhardt, 1867 coletados nos rios Cipó, Curimataí, Pardo Grande e Velhas, bacia do rio das Velhas, sudeste do Brasil, setembro de 2007, para as duas primeiras funções canônicas discriminantes.

pontos está distribuída à direita do zero, sendo apenas os escores deste rio diferente dos demais $\left(\mathrm{F}_{(156,3)}=30,65 ; \mathrm{p}<\right.$ 0,01). Em relação ao segundo eixo (PCA2), os indivíduos dos rios Cipó e Curimataí estão preferencialmente abaixo do zero e aqueles dos rios Pardo Grande e Velhas, acima do zero. Para este eixo, os grupos de escores que caracterizam cada rio diferiram entre si $\left(\mathrm{F}_{(156,3)}=22,93\right.$; $\mathrm{p}<0,01$ ), exceto Velhas e Pardo Grande.

A Análise Discriminante Canônica mostrou haver diferença significativa entre pelo menos uma das populações de $P$. argentea em relação aos atributos ecomorfológicos (Wilk's $\lambda=0,347 ; \mathrm{F}_{9,374}=22,69 ; \mathrm{p}<0,01$ ). As populações foram separadas por distâncias de Mahalanobis significativamente diferentes, sendo os pares VelhasCipó, Velhas-Curimataí e Velhas-Pardo Grande $\left(\mathrm{D}^{2}=\right.$ 11,$5 ; \mathrm{D}^{2}=5,3 \mathrm{D}^{2}=7,1$ respectivamente), os mais distantes (Fig. 7), em congruência com os resultados da PCA. Os atributos retidos no modelo e que mais contribuíram para a separação dos grupos foram, em ordem de importância, comprimento relativo da nadadeira pélvica, altura relativa do corpo e razão aspecto da nadadeira pélvica (Tab. III). Os demais atributos foram excluídos do modelo por sua baixa contribuição à separação dos grupos. A análise foi capaz de classificar corretamente $82,5 \%$ dos exemplares de $P$. argentea do rio das Velhas, $80 \%$ dos indivíduos do rio Cipó, $65 \%$ dos exemplares do rio Pardo Grande e apenas $35 \%$ do rio Curimataí.

Mesmo com as diferenças ecomorfológicas acima encontradas entre as populações dos diferentes rios, o teste de Mantel indicou não haver correlação entre ecomorfologia e uso de habitat $(r=-0,19 ; \mathrm{p}=0,99)$.

Indivíduos tenderam a ocupar habitat com menor velocidade da água nos quatro rios amostrados
(Tab. IV). Apenas no rio Cipó, exemplares maiores preferiram habitat rápidos, com velocidades entre 2,0 e $2,5 \mathrm{~m} / \mathrm{s}$. Nos demais rios, indivíduos de todos os tamanhos habitaram apenas ambientes com baixas velocidades. No rio das Velhas, $P$. argentea foi encontrada somente em velocidades entre 0 e 0,5 $\mathrm{m} / \mathrm{s}$. No rio Pardo Grande, indivíduos acima de 25 $\mathrm{cm}$ apresentaram uso de habitat dividido entre as duas primeiras classes de velocidade.

Piabina argentea foi encontrada em todas as classes de profundidade (Tab. IV), sendo que nos quatro rios, os indivíduos menores preferiram habitat rasos $(0 \mathrm{a}$ $20 \mathrm{~cm}$ no caso do rio Cipó e 20 a $40 \mathrm{~cm}$ nos demais). Nos rios Cipó, Curimataí e Velhas, os maiores indivíduos foram mais abundantes em maiores profundidades. No rio Pardo Grande, o padrão foi semelhante entre as classes de tamanho.

Tab. III. Valores de F e tolerância dos atributos ecomorfológicos e contribuição dos eixos retidos no modelo da Análise da Função Discriminante Canônica Passo-a-passo Backward para indivíduos de Piabina argentea Reinhardt, 1867 coletados nos rios Cipó, Curimataí, Pardo Grande e Velhas, bacia do rio das Velhas, sudeste do Brasil, setembro de 2007.

\begin{tabular}{lcc}
\hline Variáveis retidas no modelo & F-para-sair & Tolerância \\
\hline $\begin{array}{l}\text { Comprimento relativo da } \\
\text { nadadeira pélvica }\end{array}$ & 40,51 & 0,88 \\
$\begin{array}{l}\text { Altura relativa } \\
\text { Razão aspecto da nadadeira }\end{array}$ & 25,00 & 0,85 \\
pélvica & 15,69 & 0,90 \\
\hline & \multicolumn{2}{c}{ Eixos } \\
\cline { 2 - 3 } & DCA1 & DCA2 \\
Constante & $-5,45$ & $-3,24$ \\
Autovalor & 1,60 & 0,11 \\
Explicação cumulativa & 0,94 & 0,99 \\
\hline
\end{tabular}




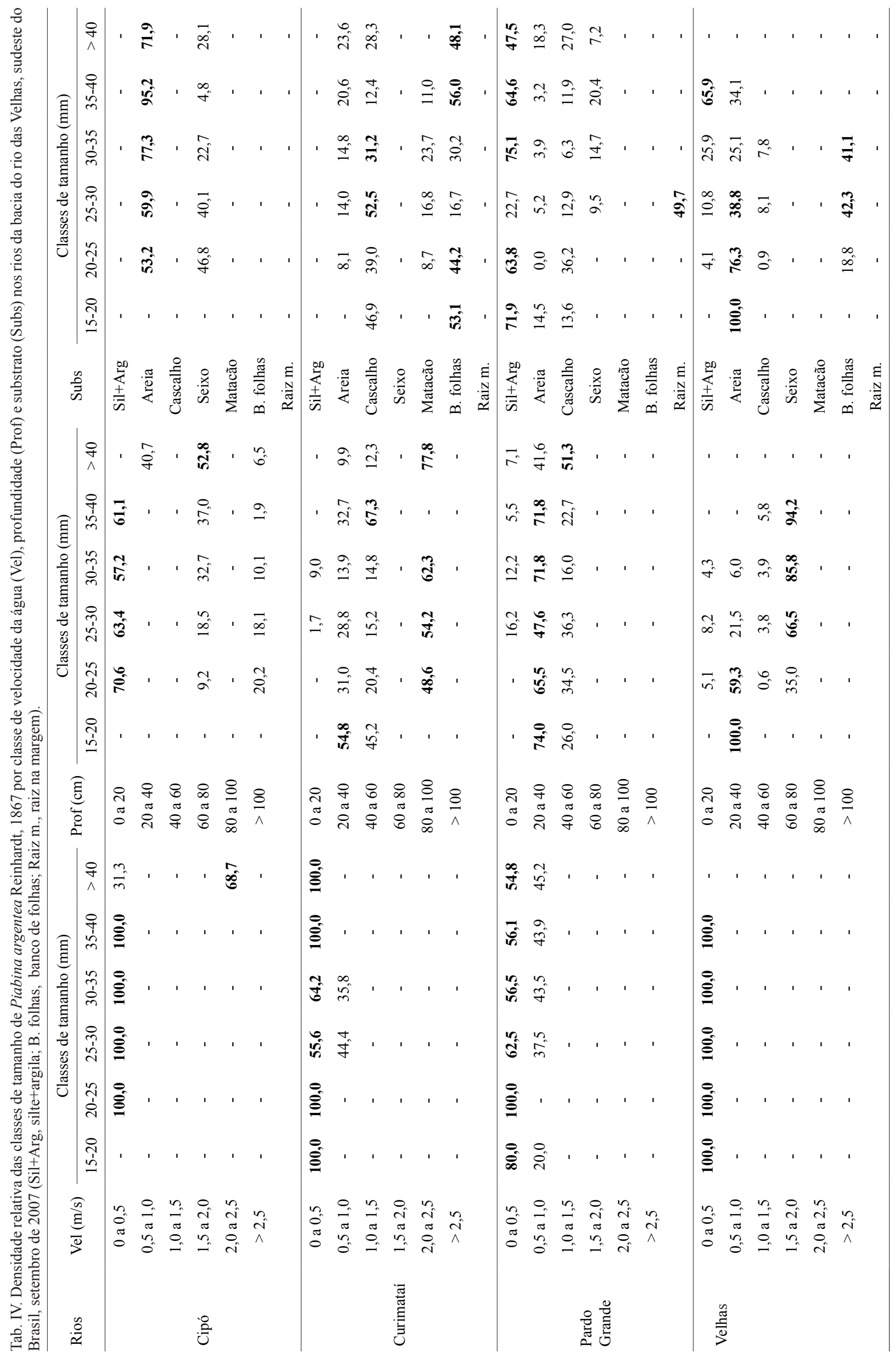


Tab. V. Densidade relativa de Piabina argentea Reinhardt, 1867 nas diferentes classes de velocidade da água, profundidade e substrato na bacia do rio das Velhas, sudeste do Brasil, setembro de 2007.

\begin{tabular}{|c|c|c|c|c|c|}
\hline Velocidade & Densidade (\%) & Profundidade & Densidade (\%) & Substrato & Densidade $(\%)$ \\
\hline $0-0,5$ & 70,0 & $0-20$ & 8,0 & Silte+argila & 22,7 \\
\hline $0,5-1,0$ & 26,7 & $20-40$ & 28,6 & Areia & 31,7 \\
\hline $1,0-1,5$ & 1,7 & $40-60$ & 7,5 & Cascalho & 10,5 \\
\hline $1,5-2,0$ & 0,0 & $60-80$ & 37,8 & Seixo & 6,7 \\
\hline $2,0-2,5$ & 1,7 & $80-100$ & 7,7 & Matacão & 7,9 \\
\hline \multirow[t]{2}{*}{$>2,5$} & 0,0 & $>100$ & 10,4 & Banco de folhas & 19,3 \\
\hline & & & & Raiz na margem & 1,2 \\
\hline
\end{tabular}

Não houve um padrão similar de uso de substrato por classe de tamanho entre os quatro rios (Tab. IV). Piabina argentea foi encontrada no rio Cipó associada apenas a dois tipos de substrato: seixo e principalmente areia em todas as classes de tamanho. No rio Curimataí indivíduos maiores e menores selecionaram banco de folhas, mas para todas as classes houve uso de áreas em cascalho. No rio Pardo Grande, a seleção foi por silte+argila em todas as classes de comprimento padrão, exceto indivíduos de 25 a $30 \mathrm{~cm}$, que preferiram raiz na margem. No rio das Velhas, exemplares de 15 a 25 $\mathrm{cm}$ foram mais frequentes em areia; de $25 \mathrm{a} 35 \mathrm{~cm}$, em banco de folhas e de 35 a $40 \mathrm{~cm}$, silte+argila.

$\mathrm{O}$ padrão regional, dos quatro rios juntos, de seleção para as populações de $P$. argentea foi de habitat com velocidade da água predominantemente mais lenta, de profundidade intermediária e substrato areia, silte+argila ou banco de folhas (Tab. V). Para profundidade e substrato, a espécie ocorreu em todo o gradiente amostrado e sua seleção foi menos marcante do que para velocidade.

\section{DISCUSSÃO}

Para $P$. argentea avaliada neste estudo, houve diferença ecomorfológica entre as populações dos quatro rios da bacia do rio das Velhas, corroborando a hipótese pressuposta. Embora estas populações tenham se sobreposto parcialmente no espaço ecomorfológico, houve diferença significativa entre elas, sobretudo do rio das Velhas em relação aos demais tributários. Os grupos de indivíduos de cada rio fazem parte de um gradiente morfológico e não são unidades discretas totalmente separadas, o que justifica a baixa porcentagem de variância explicada pela Análise dos Componentes Principais. Desta forma, nos extremos do gradiente observam-se os exemplares mais distintos.

Tanto a Análise dos Componentes Principais quanto a Análise Discriminante Canônica, destacaram aspectos das nadadeiras pélvicas como importantes atributos da separação dos grupos de $P$. argentea. Entretanto a correlação entre os atributos ecomorfológicos e as características físicas dos habitat de cada rio não foi significativa, possivelmente pelo fato de apenas o rio das Velhas ser ecomorfologicamente diferente dos demais. Da mesma forma, OLIVEIRA et al. (2010) encontraram fraca correlação entre tipo de habitat e morfologia da assembleia de peixes da planície de inundação do alto rio Paraná, mas correlação forte entre estrutura trófica e morfologia. Ainda assim, as nadadeiras pélvicas são conhecidas como responsáveis por ajustes delicados aos movimentos dos peixes (BREDA et al., 2005), indicando que em rios diferentes os requerimentos e ajustes natatórios são diferentes uma vez que a espécie pode ocupar habitat distintos. Variações nas estratégias ecológicas usadas por diferentes populações da mesma espécie são comumente observadas em uma variedade de escalas geográficas (BRINSMEAD \& Fox, 2002). Os ajustes finos à locomoção são importantes para peixes ocupando habitat lênticos, onde frequentemente realizam manobras (Neves \& MonTEIRo, 2003), como o caso de $P$. argentea.

É improvável que um único fenótipo promova o melhor desempenho para uma espécie em todas as condições ambientais as quais ela está sujeita (Pakkasmaa \& Pitronen, 2001). Embora façam parte da mesma bacia, os quatro trechos de rios estudados apresentam peculiaridades, que podem ser a causa da variação morfológica intraespecífica de $P$. argentea. $\mathrm{O}$ rio Cipó, um dos principais tributários da bacia, apresenta principalmente habitat fundos, de baixa velocidade e cobertos por seixo. O rio Curimataí diferenciou-se por apresentar diversas corredeiras e maior frequência de matacão e banco de folhas. $\mathrm{O}$ rio Pardo Grande, rico em praias de areia, possui um grande trecho formado por habitat rasos com seixo e matacão de substrato. $\mathrm{O}$ rio das Velhas é bastante assoreado, com maior velocidade da água, vegetação marginal escassa e seu principal substrato é silte+argila. As populações deste rio revelaram características associadas à ocupação de ambientes com águas rápidas e com baixa complexidade estrutural como maior achatamento ventral e maior razão aspecto das nadadeiras peitoral e pélvica, ou seja, corpo mais baixo e nadadeiras medianas mais longas do que largas, juntamente com o pedúnculo caudal mais longo (Gatz, 1979b; Watson \& Balon, 1984), o que evidencia que o ambiente está promovendo uma seleção dos exemplares mais adaptados às condições de águas mais rápidas do local.

Piabina argentea é conhecida como nectônica (CAStro et al., 2004), diurna e onívora (Ferreira, 2007). CASATTI et al. (2006) apontam ainda que a espécie é uma potencial indicadora de habitat preservados, como observado no sistema do rio São José dos Dourados, 
sudeste do Brasil. Na bacia do rio das Velhas, localidade tipo da $P$. argentea, esta espécie possui distribuição ampla (Alves \& POMPeU, 2001) e, no presente estudo, foi encontrada tanto em tributários bem preservados, quanto no trecho degradado do rio, onde foi mais abundante. Os eixos do PCA mostraram separação de $P$. argentea em relação ao caráter bentônico ou nectônico dos indivíduos e ocupação de águas turbulentas (PCA1) e também em relação à velocidade da água (PCA2). Desta forma, os indivíduos do rio das Velhas apresentam caráter nectônico e atributos relacionados a habitat turbulentos, enquanto nos tributários, os exemplares foram principalmente bentônicos. Apesar do conhecido hábito nectônico, FERREIRA (2007) observou que a espécie é onívora com tendência à insetivoria e possui grande plasticidade alimentar, podendo capturar presas tanto na coluna d'água quanto no substrato. $\mathrm{O}$ assoreamento do rio das Velhas, possivelmente the confere um substrato de menor riqueza e abundância de presas, levando $P$. argentea a explorar recursos apenas na coluna d'água. A espécie não apresenta adaptações morfológicas estritamente ligadas ao hábito bentônico, mas nos tributários, presume-se que a população esteja mais adaptada ao forrageamento tanto da coluna d'água quanto junto ao substrato.

A velocidade da água é amplamente citada como um importante fator que pode promover divergência morfológica em peixes, principalmente em relação à ocupação de habitat lóticos e lênticos (PAKKASMAA \& Pirronen, 2001; Brinsmead \& Fox, 2002; LANGERHANS et al., 2003; Neves \& Monteiro, 2003). Os esforços do escoamento da água sobre o corpo dos peixes tem alta correlação com aspectos hidrodinâmicos (MATTHEws, 1998) e devem limitar a ocupação dos habitat por determinados tipos morfológicos. No caso de P. argentea, os quatro rios estudados têm o mesmo caráter lótico, embora apresentem diferenças relativas à quantidade de habitat de águas rápidas ou mais lentas. O grupo de indivíduos do rio das Velhas foi morfologicamente mais distinto das populações dos tributários do que estas entre si. Indicando que o ambiente selecionou indivíduos dentro da população que são mais adaptados às condições locais do rio das Velhas. Esse resultado pode também ser devido à maior distância da população do rio das Velhas, localizada no alto curso da bacia, dos tributários, situados em seu médio curso e mais próximos entre si. O fluxo gênico entre populações é uma restrição à divergência morfológica (LENORMAND, 2002; LANGERHANs et al., 2003). Considerando apenas os rios referência, os indivíduos do rio Curimataí, que apresentou menor porcentagem de classificação correta dos casos a partir da função discriminante, foram mais semelhantes aos dos rios Cipó e Pardo Grande, do que estes entre si. Caracteres morfológicos são raramente constantes dentro das espécies, sendo que algumas apresentam pequena variação fenotípica, outras variam em maior escala em populações diferentes (Travis, 1994).
Da mesma forma, a velocidade da água é destacada como um dos principais aspectos físicos da preferência de habitat dos peixes (JowetT \& Richardson, 1995; Lamouroux et al., 1999). Piabina argentea foi mais restritiva à velocidade média da água e mais generalista aos outros aspectos físicos dos habitat. A seleção por habitat com maior velocidade da água foi congruente entre rios e classes de tamanho. Já em relação à profundidade e ao substrato, a espécie pareceu mais generalista. Considerando as classes de tamanho, indivíduos de $P$. argentea de menor tamanho apresentaram seleção de habitat mais marcada, sendo mais restritivos às condições de velocidade e profundidade. Em todos os rios indivíduos menores utilizaram habitat lentos e rasos, sendo locais rasos mais adequados à proteção contra predadores de maior porte, e locais lentos, menos severos aos peixes por requererem menor gasto energético para locomoção (CASATTI \& CASTRO, 2006), refletindo a menor capacidade natatória desses indivíduos.

Identificar processos locais comuns a diferentes regiões é uma etapa importante para definir ferramentas de manejo aplicáveis em maior escala (LAMOURoux et al., 1999). Os padrões locais de seleção de habitat de Piabina argentea não foram congruentes em todos os rios, mas em geral, houve predomínio do padrão regional. Um corpo d'água assoreado como o trecho rio das Velhas, tende a ter seu leito mais homogêneo em termos de diversidade de habitat, maiores velocidades da água, menores profundidades e predominância de substrato fino. Isso atende em parte ao uso de habitat de $P$. argentea, principalmente a maior velocidade da água. A espécie ainda é encontrada em grande densidade e ampla distribuição no rio das Velhas, sugerindo que a atual condição de degradação da bacia não esteja interferindo em sua distribuição. Entretanto, com o agravamento do atual processo de assoreamento, pode tornar-se cada vez mais restrita a determinados habitat neste trecho e também em outras áreas degradadas da bacia.

Agradecimentos. Agradecemos a Carlos Bernardo M. Alves, Lucas V. Pires, Miriam Aparecida de Castro, Ceceo Chaves, Stephanie F. Cunha, Ivo A. Figueiredo, Paulo Pinheiro, Fernanda Horta, Marianne Antunes, Juliana França e Lurdemar Tavares, pela participação no trabalho de campo; Lucas V. Pires e Sara Saraiva pelo auxílio na obtenção dos atributos ecomorfológicos e Daniel N. Rodrigues pela elaboração do mapa; a Lilian Casatti, Fábio Vieira e dois revisores anônimos pelas valiosas sugestões ao manuscrito; ao Projeto Manuelzão e a Nuvelhas (Núcleo Transdisciplinar e Transinstitucional para Revitalização da Bacia do Rio das Velhas) pelo apoio logístico. Este estudo foi financiado pelo CNPq (Programa CTHidro, Proc. 555698/2006-8) e a FAPEMIG concedeu bolsa de mestrado para CGL.

\section{REFERÊNCIAS BIBLIOGRÁFICAS}

Allen, M. A. 2000. Seasonal microhabitat use by juvenile Spring Chinook Salmon in the Yakima River Basin, Washington. Rivers 7(4):314-332.

Alves, C. B. M. \& Pompeu, P. S. 2001. A fauna de peixes da bacia do rio das Velhas no final do século XX. In: Alves, C. B. M. \& Pompeu, P. Dos S. orgs. Peixes do rio das Velhas: passado e presente. Belo Horizonte, SEGRAC. p. 165-187. 
2005. Historical changes in the rio das Velhas fish fauna Brazil. American Fisheries Society Symposium 45:587-602.

Blanck, A.; Tedesco, P. A. \& Lamouroux, N. 2007. Relationships between life-history strategies of European freshwater fish species and their habitat preference. Freshwater Biology 52:843-859.

Breda, L.; Oliveira, E. F. \& Goulart, E. 2005. Ecomorfologia de locomoção de peixes com enfoque para espécies neotropicais. Acta Scientiarum, Biological Sciences 27(4):371-381.

BRINSMEAD, J. \& Fox, M. G. 2002. Morphological variation between lake- and stream-dwelling rock bass and pumpkinseed populations. Journal of Fish Biology 6:1619-1638.

Callisto, M. \& Esteves, F. 1996. Composição granulométrica do sedimento de um lago amazônico impactado por rejeito de bauxita e um lago natural. Acta Limnologica Brasiliensia 8:115-126.

Casatti, L. \& CAstro, R. M. C. 2006. Testing the ecomorphological hypothesis in a headwater riffles fish assemblage of the rio São Francisco, southeastern Brazil. Neotropical Ichthyology 4(2):203-214

Casatti, L.; Langeani, F. \& Ferreira, C. P. 2006. Effects of physical habitat degradation on the stream fish assemblage structure in a pasture region. Environmental Management 38:974-982.

Castro, R. M. C.; Casatti, L.; Santos, H. F.; Melo, A. L. A.; Martins, L. S. F.; Ferreira, K. M.; Gibran, F. Z.; Benine, R. C.; Carvalho, M.; Ribeiro, A. C.; Abreu, T. X.; Bockmann, F. A.; Pelição, G. Z.; Stopiglia, R. \& LangeAni, F. 2004. Estrutura e composição da ictiofauna de riachos da bacia do rio Grande no estado de São Paulo, sudeste do Brasil. Biota Neotropica 4(1):1-39.

CETEC. 1983. Diagnóstico ambiental do estado de Minas Gerais. Belo Horizonte, Fundação Centro Tecnológico de Minas Gerais (Série de Publicações Técnicas). 158p.

CODEVASF. 1986. PLANVASF: Plano diretor para o desenvolvimento do Vale do São Francisco: síntese. Brasília, Companhia de Desenvolvimento do Vale do São Francisco. 80p.

Cunico, A. M. \& Agostinho, A. A. 2006. Morphological Patterns of Fish and Their Relationships with Reservoirs Hydrodynamics. Brazilian Archives of Biology and Tecnology 49(1):125-134

FerreIRA, K. M. 2007. Biology and ecomorphology of stream fishes from the rio Mogi-Guaçu basin, Southeastern Brazil. Neotropical Ichthyology 5(3):311-326.

Freire, A. G. \& Agostinho, A. A. 2001. Ecomorfologia de oito espécies dominantes da ictiofauna do reservatório de Itaipu (Paraná/Brasil). Acta Limnologica Brasiliensia 13(1):1-9.

Gatz, A. J. JR.1979a. Community organization in fishes as indicated by morphological features. Ecology 60:711-718.

1979b. Ecological morphology of freshwater stream fishes. Tulanne Studies in Zoology and Botany 21:91-124.

GORMan, O. T. \& KarR, J. R. 1978. Habitat structure and stream fish communities. Ecology 59(3):507-515.

Gosline, W. A. 1971. Functional morphology and classification of teleostean fishes. Honolulu, University Press of Hawaii. 208p

Grossman, G. D. \& De SostoA, A. 1994. Microhabitat use by fish in the lower Rio Matarranã, Spain, 1984-1987. Ecology of Freshwater Fish 3:123-136.

Grossman, G. D.; RatajczaK Júnior, R. E.; Crawford, M. \& FreEMAN, M. C. 1998. Assemblage organization in streams fishes: effects of environmental variation and interespecific interactions. Ecological Monographs 68(3):395-420.

Guill, J. M.; Hood, C. S. \& Heins, D. C. 2003. Body shape variation within and among three species of darters (Perciformes: Percidae). Ecology of Freshwater Fish 12:134-140.

Hora, S. L. 1930. Ecology, bionomics and evolution of the torrential fauna, with special reference to the organs of attachment. Philosophical Transactions of the Royal Society of London 28:171-282.

JOWETT, I. G. \& RiCHARDSON, J. 1995. Habitat preference of common, riverine New Zealand native fishes and implications for flow management. New Zealand Journal of Marine and Freshwater Research 29:13-23.

Lamouroux, N.; Capra, H.; Pouilly, M. \& Souchon, Y. 1999. Fish habitat preferences in large streams of southern France. Freshwater Biology 42(4):673-687.
Langerhans, R. B.; Layman, C. A.; Langerhans, A. \& Dewitt, T. J. 2003. Habitat-associated morphological divergence in two Neotropical fish species. Biological Journal of the Linnean Society 80:689-698.

Leal, C. G.; Jungueira, N. T. \& Pompeu, P. S. 2011. Morphology and habitat use by fishes of the Rio das Velhas basin in southeastern Brazil. Environmental Biology of Fishes 90:143-157.

LenORMAND, T. 2002. Gene flow and the limits to natural selection. Trends in Ecology and Evolution 17:183-189.

Lima, F. C. T.; Malabarba, L. R.; Buckup, P. A.; Silva, J. F. P.; Vari, R. P.; Harold, A.; Benine, R.; Oyakawa, O. T.; Pavanelli, C. S.; Menezes, N. A.; Lucena, C. A. S.; Malabarba, M. C. S L.; Lucena, Z. M. S.; Reis, R. E.; Langeani, F.; Casatti, L.; Bertaco, V. A.; Moreira, C. \& Lucinda, P. H. F. 2003. Genera incertae sedis in Characidae. In: ReIs, R. E.; Kullander, S. O \& FERraris Jr., C. J. eds. Check list of the freshwater fishes of South and Central America. Porto Alegre, Edipucrs. p.106 169.

Lowe-McConnell, R. H. 1987. Ecological studies in tropical fish communities. Cambridge, Cambridge University Press. 379 p.

Matthews, W. J. 1998. Patterns in Freshwater Fish Ecology. New York, Chapman and Hall. 756p.

Neves, F. M. \& Monteiro, L. R. 2003. Body shape and size divergence among populations of Poecilia vivipara in coastal lagoons of south-eastern Brazil. Journal of Fish Biology 63:928-941.

Oliveira, E. F.; Goulart, E.; Breda, L.; Minte-Vera, C. V.; Paiva, L. R DE S.\& VISMARA, M. R. 2010. Ecomorphological patterns of the fish assemblage in a tropical floodplain: effects of trophic, spatia and phylogenetic structures. Neotropical Ichthyology 8(3):569586.

PakkasmaA, S. \& PiIronen, J. 2001. Water velocity shapes juvenile salmonids. Evolutionary Ecology 14:721-730.

Pompeu, P. S.; Callisto, M. \& Alves, C. B. M. 2005. The effects of urbanization on biodiversity and water quality in the Rio das Velhas basin, Brazil. American Fisheries Society Symposium 47:11-22.

R Development Core Team. 2012. R: A language and environment for statistical computing. $\mathrm{R}$ Foundation for Statistical Computing. Disponível em: <http://www.R-project.org/>. Acesso em: 30.10 .2012 .

Robinson, B. W. \& WiLson, D. S. 1994. Character release and displacement in fishes: a neglected literature. American Naturalist 144:596-627.

Rosenfeld, J. 2003. Assessing the habitat requirements of stream fishes: an overview and evaluation of different approaches. Transactions of American Fisheries Society 132:953-968.

Smith, T. B. \& Skúlason, S. 1996. Evolutionary significance of resource polymorphisms in fishes, amphibians, and birds. Annual Review of Ecology and Systematics 27:111-133.

Statsoft, InC. 2001. Statistica for Windows (Computer program manual). Disponível em: <www.statsoft.com>. Acesso em: 03.01.2008

Suguio, K. 1973. Introdução à sedimentologia. São Paulo, Edgard Blücher. 317p.

Travis, J. 1994. Evaluating the adaptative role of morphological plasticity. In: WaINWRight, C. P. \& ReILly, S. M. eds. Ecological Morphology: integrative organismal in biology. Chicago, University of Chicago Press. p.99-122.

VADAS JúNIOR, R. L. \& ORTH, D. J. 2000. Habitat use of fish communities in a Virginia stream system. Environmental Biology of Fish 59:253-269.

Wainwright, P. C. 1994. Functional morphology as a tool in ecological research. In: WaInWRight, C. P. \& ReILly, S. M. eds. Ecological Morphology: Integrative Organismal Biology. Chicago, University of Chicago Press. p.42-59.

WATSON, D. J. \& BALON, E. 1984. Ecomorphological analysis of taxocenes in rainforest streams os horthern Borneo. Journal of Fish Biology 25:371-384.

Winemiller, K. O. 1991. Ecomorphological diversification in lowland freshwater fish assemblages from five biotic regions. Ecological Monographs 61:343-365

Recebido em 31 de outubro de 2012. Aceito em 22 de agosto de 2013. ISSN 0073-4721

Artigo disponível em: www.scielo.br/isz 